\title{
The HL7 RIM in the Design and Implementation of an Information System for Clinical Investigations on Medical Devices
}

\author{
Daniela Luzi ${ }^{1}$, Mariangela Contenti ${ }^{2}$, and Fabrizio Pecoraro ${ }^{1}$ \\ ${ }^{1}$ National Research Council, Institute for Research on Population and Social Policy, \\ Via Palestro, 32, I-00100 Rome, Italy \\ \{d.luzi,f.pecoraro\}@irpps.cnr.it \\ ${ }^{2}$ National Research Council, Institute for Biomedical Technologies, \\ Circonvallazione Nomentana, 496, I-00100 Rome, Italy \\ m.contenti@itb.cnr.it
}

\begin{abstract}
The paper describes MEDIS (Medical Device Information System), a system developed to support the whole lifecycle of a Clinical Investigation (CI) for Medical Devices, providing details on the approach used in its development. MEDIS is a software system that collects and manages data and documents, exchanged between an applicant and the Italian National Competent Authority during all the phases of the business process, from the CI notification up to the submission of the final results. The development of the MEDIS Domain Analysis Model (DAM) following the HL7 v.3 methodology as well as the approach followed in the system implementation are discussed in relation to the business domain peculiarities.
\end{abstract}

Keywords: Information Model, HL7, Clinical Investigation, Medical Devices.

\section{Introduction}

Medical devices (MDs) are becoming ever increasingly used tools in daily clinical practice to diagnose as well as treat or prevent many diseases. Their development and final product commercialization undergo several steps, which generally include their testing and assessment during Clinical Investigations (CIs), where MDs' safety and efficacy are verified under normal condition of use.

European directives [1], national laws as well as a body of international technical norms regulate CIs approval procedures and performances. At European level the aim is to implement a common Member States approach able both to facilitate MD manufacturers to perform international multi-centric investigations, and to improve information exchange among National Competent Authorities (NCA) that evaluate CI proposals and monitor their performance. EUDAMED (European Database for Medical Devices) is following these objectives, currently focusing on information sharing related to MDs placed on the market, while the design of a European registry on CIs of MDs is in its early stage. 
To improve communication, information sharing on MDs has to be based on the identification of a standard set of data able to describe MD characteristics and track them in interoperable information systems. This makes it necessary to develop a common standard language to achieve semantic interoperability among systems and organizations.

In Italy the National Research Council is carrying out a project supported by the Ministry of Health aiming to develop an information system - MEDIS (Medical Device Information System) - that manages the information flows between applicants and the Ministry of Health's Competent Authority. MEDIS plays the role of both a registry of clinical investigation data and a content repository of documents submitted by manufacturers to the NCA to obtain the approval for the clinical investigation start. In particular, MEDIS supports manufacturers in the documentation submission process as well as the NCA evaluators in assessing the data received. It also manages the communication among the different stakeholders and collects the data produced during the whole lifecycle of clinical investigations.

The paper describes the MEDIS system, providing details on the approach used in its development. The adoption of the HL7 v.3 methodology is explained on the basis of the business domain characteristics. After the description of the clinical investigation lifecycle, we analyze standardization initiatives related to clinical investigation for medical devices. Afterwards a concise description of the MEDIS system is given. We describe our approach providing some examples of the MEDIS DAM related to its transformation to a data model.

\section{The Clinical Investigation Lifecycle}

A high level description of the lifecycle of a CI on MDs is shown in figure 1 using BPMN notation. The business process can be divided into three main sub-processes:

- In the notification sub-process the CI applicant sends the documentation composed by administrative documents (letter of designation of an authorized representative, signed statement, etc.) and technical documentation (clinical protocol, the risk analysis document, etc.) to the NCA.

- In the evaluation sub-process, the NCA activates the administrative procedure assigning the notification to an evaluation team. Its tasks are to verify the formal completeness and consistency of the documentation submitted and to analyze its content in order to assess MD safety requirements as well as the scientific, clinical and ethical fulfillments of the clinical protocol. During this sub-process the NCA can require further information and documents.

- If the CI proposal is approved, the investigation sub-process can begin and its start date is communicated by applicants to the NCA. During this sub-process, the applicant has also to notify amendments in the clinical protocol, if any, and/or report serious adverse events that may have occurred during the CI. In any case, end date of the investigation or eventually its anticipated termination has to be communicated and the final report on CI results provided. 
The HL7 RIM in the Design and implementation of an Information System

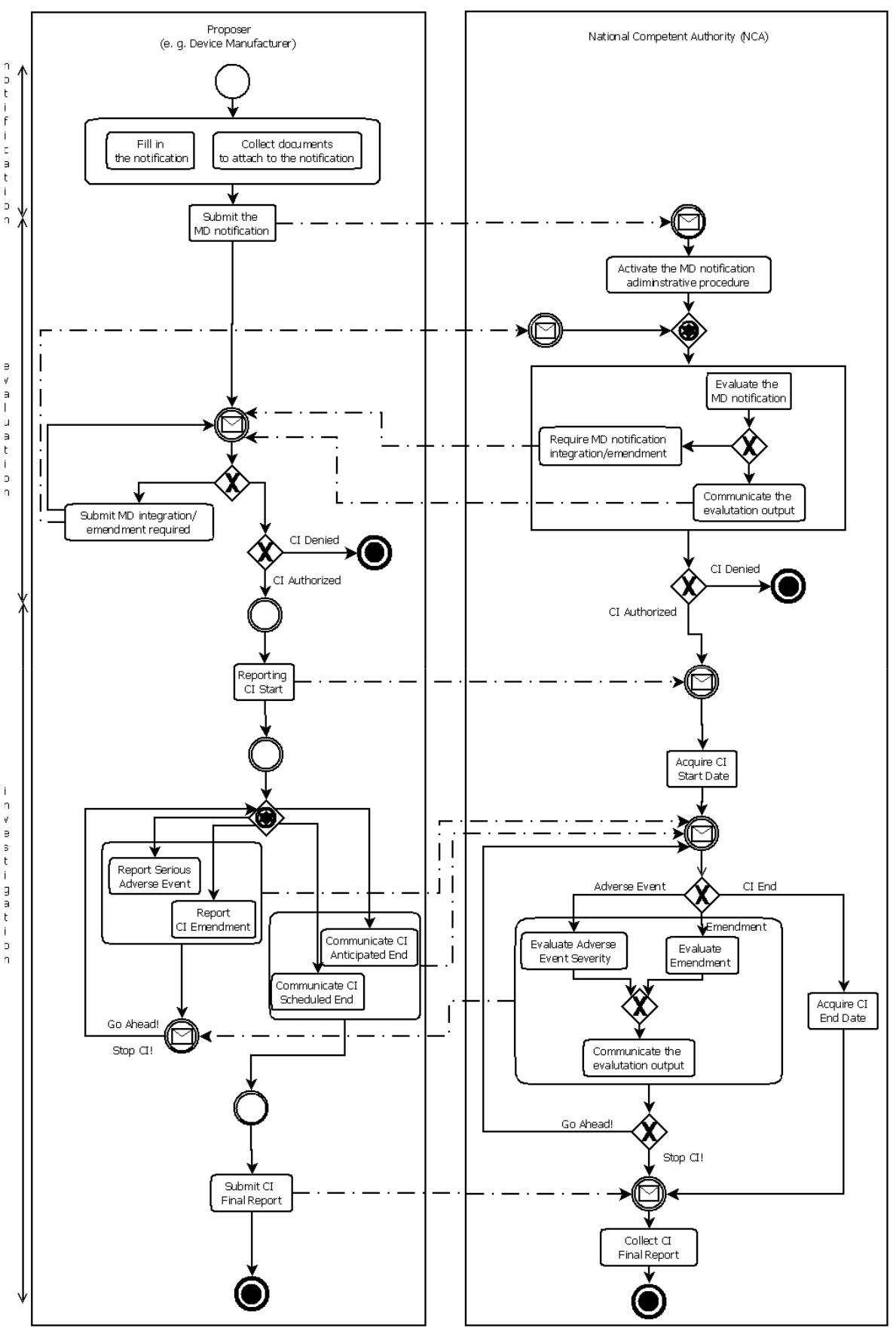

Fig. 1. The clinical investigation lifecycle 
The majority of the activities carried out in the business process pertain to a communication exchange between CI applicants and NCA. Moreover, other participants could be included in the messaging flow, such as Ethical Committees, that have to approve the CI verifying its scientific merits and ethical acceptability, and clinical investigators, who could directly report information on Serious Adverse Events.

\section{Standardization Initiatives}

Even if European Directives provide the main requirements for CI approval, there are still many differences concerning the types of data and documents required by NCAs. In Europe only few countries have developed their own electronic submission system of CIs on MDs, the majority of them still relies on a paper-based information exchange. Among the organizations that develop health care standards, it is worth mentioning Clinical Data Interchange Standard Consortium (CDISC) and Health Level Seven (HL7). These organizations have begun to standardize the description of MDs capturing aspects related to specific points of view and/or sub-processes. However, a comprehensive standardized model pertaining MDs within the CI framework is still missing.

CDISC is developing standards to support the acquisition, exchange, submission and archive of clinical research data and metadata, focusing in particular on clinical trials on pharmaceutical products [2]. Recently a SDTM (Study Data Tabulation Model) Device sub-team has been formed with the aim of developing a domain that describes information (properties and characteristics) usable for different types of devices such as implantable devices, imaging and diagnostic devices. Starting from the SDTM related to clinical trials on pharmaceuticals products, the sub-team is proposing a device property domain, able to capture MDs data and metadata collected by manufacturers during CIs [3].

Health Level Seven (HL7) provides standards for the exchange, management and integration of electronic health care related data. It has released three models focusing on particular aspects of the MD domain $[4,5]$. One was created by the "Implantable Device-Cardiac" Project within the Therapeutics Device domain, which comprises models, messages related to cardiac therapy delivery and observations related to implantable medical devices. Another standard concerns the Regulated Product Domain, where MDs are described in the framework of adverse events to be tracked in surveillance processes. Finally, the Regulated Studies Domain describes a part of clinical investigation through the Periodic Reporting of Clinical Trial Laboratory Results.

Also the joint effort carried out by BRIDG (Biomedical research Integrated Domain Group) [6] and caBig (cancer Biomedical Informatics Grid) [7], considers the domain of clinical trial on pharmaceutical products, focusing on the harmonization between HL7 messaging model and CIDSC data model.

\section{MEDIS System}

MEDIS is a registry that collects data and documents exchanged between applicants and NCA during the whole lifecycle of a CI, from the phase of notification to the phase of collecting the final results. The system supports: 
- Applicants in the notification submission process, i.e. the collection and submission of regulatory data and documents to the NCA in order to obtain the approval of the CI start-up as required by national and European Directives;

- NCA evaluators during the assessment of the submitted data and documents in order to authorize or deny the start-up of the proposed CI;

- Both applicants and NCA facilitating their information exchange during the whole lifecycle of a CI (e.g. request for data and/or document integration, reports of amendments and/or serious adverse events).

By collecting the data and documents uploaded throughout the whole CI, the MEDIS systems also supports NCA in scheduling the evaluation activities, as well as monitoring the ongoing $\mathrm{CI}$ performance.

From the architectural point of view, MEDIS is a client-server three-tiered system where the front-end is implemented with a mix of server-side and client-side technologies. The business logic layer consists of a set of software modules in charge of:

- Dynamic generation of electronic forms depending on the user, the MD under investigation, and the state reached in the workflow;

- Control of the completeness and consistency of the data and documents uploaded in a single form or in a correlated set of forms;

- Provision of common services (e.g. authentication and authorization, XML and PDF documents generation, digital signature, etc.).

Finally, the MEDIS persistence layer is divided into:

- A content repository in charge of archiving documents uploaded as attachments to the notification or generated by the system starting from the data filled in the electronic forms;

- A relational database that contains data describing $\mathrm{MD}$ and $\mathrm{CI}$ instances, data tracking the CI lifecycle workflow and metadata related to documents stored in the content repository.

\section{MEDIS Domain Analysis Model}

HL7 is today mainly applied within health care organizations, where different legacy systems have to interact in order to exchange administrative and clinical data on patients and health care services provided. At the basis of HL7 v.3 messaging there are three main information models:

- The Reference Information Model (RIM), which is a simple abstract framework that addresses wildly heterogeneous clinical data described through six base classes [8];

- The Domain Analysis Model (DAM), which is a specification of the RIM to define information elements for a specific domain;

- The Refined Message Information Model (R-MIM), which is a refined view of the DAM to define the information elements of a specific message or a family of messages. 
The advantages of using the HL7 RIM abstract model to define a specific domain model following the further steps to derive encoded messages make the information flows among different information systems homogeneous and sound. Moreover, the agreement reached on the DAM constitutes a common representation shared by the domain stakeholders. Therefore, the development of a MD DAM could foster already at an initial stage a common agreed data model and messaging that facilitate information sharing and seamless integration among different organizations. Following the first step of the HL7 v.3 methodology figure 2 depicts the portion of the MEDIS DAM [9] modeling MD in the framework of CI. This example shows how the HL7 DAM notation (UML class diagram notation) is able to capture important aspects of MD static and dynamic characteristics.

In our interpretation, the HL7 RIM can be decomposed into two parts:

- The static part is captured by the classes Entity and Role, i.e. objects or subjects involved in a process and the role played by them (i.e. a person who has the role of a physician);

- The dynamic part is represented by the classes Participation and Act, which describe actions, performed or scheduled, and how subjects and objects take part in them (i.e. in the activity of performing a clinical investigation a physician participates as a principal investigator).

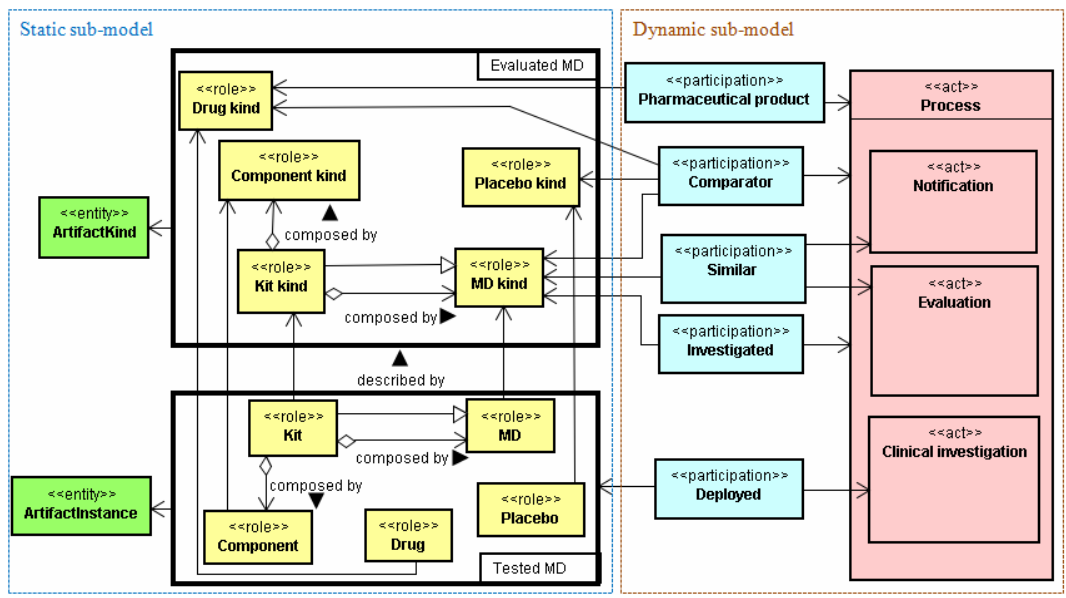

Fig. 2. MD portion of the MEDIS DAM

\subsection{Static Representation}

The regulatory documents submitted for CI approval contain detailed descriptions of the investigational MD, including MD components and their interactions, materials used, methods for its sterilization, etc. This information makes it possible to evaluate MD safety requirements as well as the risk analysis performed by the MD manufacturer. 
When a MD is actually used in the investigation, other information become relevant. This is for instance the case when it is necessary to track a specific MD implanted in a specific patient. For this reason in the DAM we model each product under two points of view: ArtifactKind and ArtifactInstance:

- The former represents an abstract description of any MD as reported in detail in the technical documents submitted to obtain the approval of the clinical investigation start-up;

- The latter models the description of a single tangible MD used in real investigation conditions.

In this way it is possible to manage data representing any medical device artifact as well as data gathered during the investigating process of a specific MD tracked by its lot or serial number. To connect these different aspects of the MD description in MEDIS DAM the attribute Determiner code of the HL7 Entity class is used. The abstract description of any product is represented by the $<<$ entity $>>$ ArtifactKind and associated with the relevant Role: MD kind, Kit kind, Component kind, Drug kind, Placebo kind (fig. 2).

\subsection{Dynamic Representation}

Many different MDs are involved in the CI lifecycle, other than the one under investigation. Some MDs often represent a technological evolution of already commercialized devices. They are often mentioned in the documentation to prove through the similarity with the investigational product the fulfillments of safety requirements. Moreover, in randomized CIs, a comparator is used to test differences in safety and efficacy between two devices.

In order to distinguish the aim and functions of these different MDs described and/or used during the CI lifecycle, we use the stereotypes Participation and Act. For instance, the Role $M D$ kind participates to the whole CI process either as Investigated or Comparator. In addition, it could participate to the Notification and Evaluation activities as Similar. Likewise, a drug can participate, as a Comparator or as a Pharmaceutical product in the whole CI process. Analogously the Roles related to ArtifactInstance are associated only to the Clinical Investigation activity through the Participation Deployed.

\section{MEDIS Data Model}

In the HL7 methodology the construction of a DAM is intended to capture in a formal language both the static and dynamic aspects of a certain domain. The sharing of a common representation of the world facilitates the communication among different parties. Actually when the interaction among parties is modeled through use cases, the HL7 Development Framework provides the methodology and tools to derive the message format through which different information systems could send and receive syntactic and semantic interoperable information. Even if the task of defining a shared format for the messages to be exchanged is simplified, when legacy systems have to interoperate, the mapping between the message format and the internal representation of data could demand a meaningful effort. 


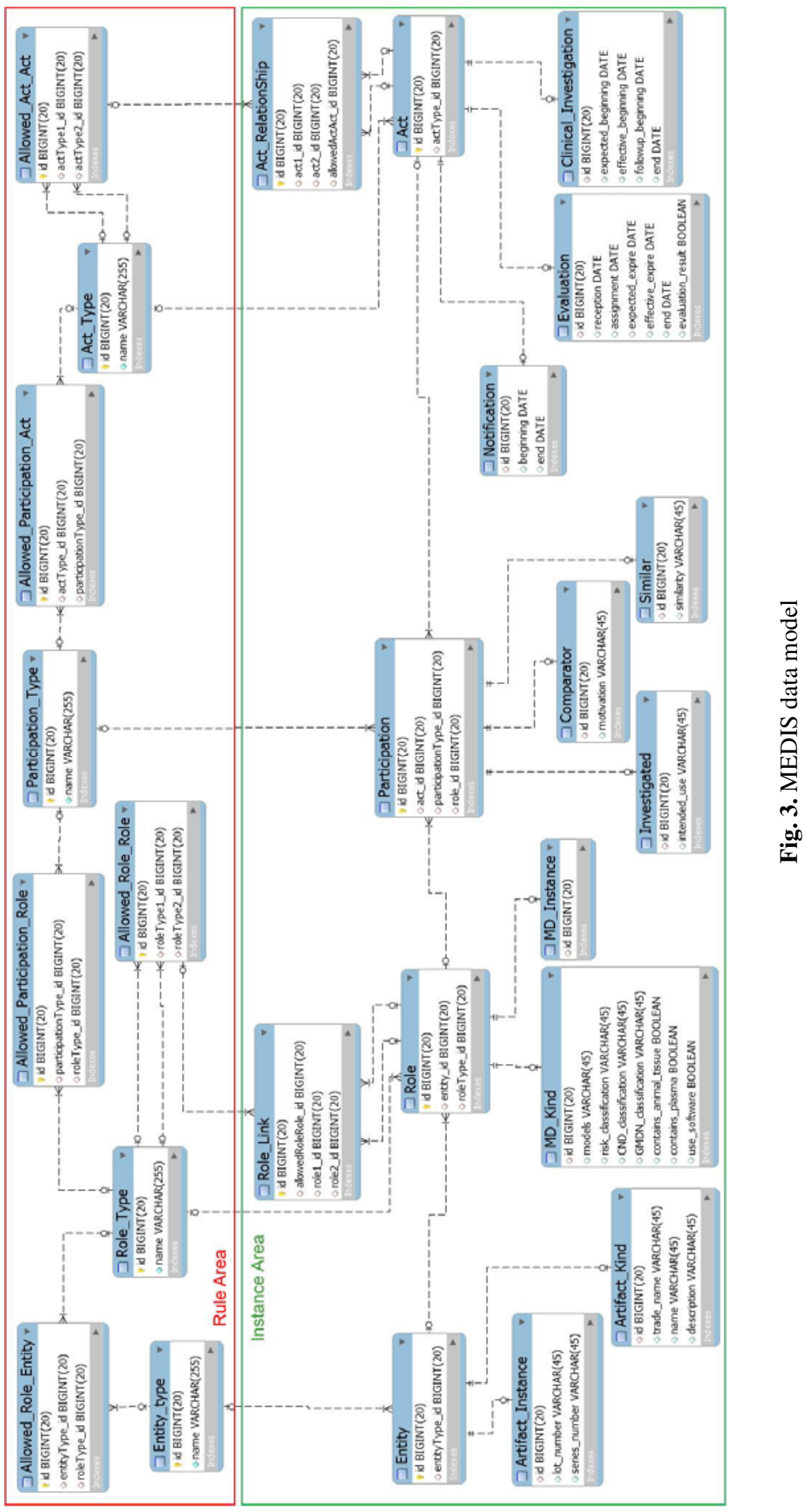


Actually some contributions trying to design and implement automation tools for mapping between DAM classes with relational databases are already available in the literature $[10,11]$.

In the MD domain there are not so many legacy systems to integrate; large part of the information systems to automate this domain still have to be developed. In this respect, our intention is to propose a normalized data model for a relational database, representative for the MEDIS DAM, in order to make the population of message instances straightforward.

The MEDIS DAM representative data model is depicted in figure 3. It is divided in two different conceptual areas, the instance and the rule areas:

- The instance area matches the MEDIS DAM and is related to the instance of the MD domain. It represents the data collected during the whole clinical investigation process.

- The rule area represents the rules of the business process of the clinical investigation life cycle semi automated by the MEDIS system.

A peculiarity of the presented relational database model is the explicit representation of the six base entities derived from the HL7 RIM meta-model. They are introduced in the instance area as generalizations of more specialized MEDIS DAM entities. They are also used for describing the rules used by the MEDIS system to determine each steps in the evolution of the workflow of any single CI.

Looking at the instance area of the database, the individual activities related to the three clinical investigation sub-processes are stored in the Notification, Evaluation and Clinical Investigation tables. The advances in the business process are described through relationship between these activities and are stored in the Act_Relationship table. In this way all the sub-processes of the clinical investigation lifecycle are linked together and in any time it is possible to identify the state reached by each CI instance.

A pre-defined set of CI activities are stored in the table Act_Type, tuples representing pre-defined consecutive activities are stored in the table Allowed_Act_Act. These rules are used by the business logic layer of the MEDIS system to determine, at any time, the sole active functionalities. This makes it possible for instance to prevent CI applicant to notify any serious adverse event before he/she has communicated the start date of the investigation.

\section{Conclusions and Future Works}

The paper describes the MEDIS system, providing details on the approach used in its development. The adoption of the HL7 v.3 methodology is explained on the basis of the business domain characteristics.

Interesting aspects of the implemented solution are also described, highlighting the co-presence in the E-R data model of the entities derived from both the specialized DAM and the abstract RIM. In particular, due to the fact that the database collects not only the CI lifecycle instances data but also the rules governing the CI lifecycle workflow, the RIM entities were introduced in order to relate the two datasets. 
Currently we are validating the MEDIS system involving real users with the objectives to evaluate the performances of the implemented solution.

In more general terms the results achieved with the development of MEDIS represent an important contribution to foster the debate among Member State on agreed upon standardization initiatives on CIs on MDs. Actually large agreement on the MEDIS DAM could facilitate the development of national registries that need to exchange information on CIs, as well as their interoperability with EUDAMED.

In this respect we are analyzing types of information exchanged between NCAs in order to identify cooperative scenarios as well as an initial set of contents, which need to be exchanged among enrolled parties. The proper modeling of real use cases in order to get the R-MIM and the interoperable message format is in its early stage.

\section{Acknowledgments}

This study was supported by the Italian Ministry of Health through the MEDIS project (MdS-CNR collaboration contract $\left.n^{\circ} 1037 / 2007\right)$.

\section{References}

1. European Parliament, Directive 2007/47/EC of the European Parliament and of the Council of 5 September 2007 amending Council Directive 90/385/EEC on the approximation of the laws of the Member States relating to active implantable medical devices, Council Directive 93/42/EEC concerning medical devices and Directive 98/8/EC concerning the placing of biocidal products on the market. Official Journal of the European Union, L. 247/21 (21.9.2007)

2. CDISC: Clinical Data Interchange Standards Consortium, http: / / www . cdisc . org

3. Smoak, C.: CDISC for the Medical Device and Diagnostic Industry: An Update (2009), http: / / www . wuss . org/proceedings09/09WUSSProceedings / papers / cdi/CDI-Smoak.pdf

4. Health Level Seven v3, http://h17.org/v3ballot/html/welcome/environment/index.htm

5. Health Level Seven, Inc. HL7 Reference Information Model. Ann Arbor, MI, http: / / www.h17.org

6. Fridsma, B.D., Evans, J., Hastak, S., Mead, C.N.: The BRIDG Project: A technical report. JAMIA 15, 130-137 (2007)

7. caBIG: Cancer Biomedical Informatics Grid, http: / / cabig.nci.nih.gov

8. Schadow, G., Mead, C.N., Walker, D.M.: The HL7 Reference Information Model under scrutiny. SHTI 124, 151-156 (2006)

9. Luzi, D., Pecoraro, F., Mercurio, G., Ricci, F.L.: A medical device domain analysis model based on HL7 RIM. STHI 150, 162-166 (2009)

10. Eggebraaten, T.J., Tenner, J.W., Dubbels, J.C.: A health-care data model based on the HL7 Reference Information Model. IBM Journal of Research and Development 46(1), 5-18 (2007)

11. Umer, S., Afzal, M., Hussain, M., Ahmad, H.F., Latif, K.: Design and implementation of an automation tool for HL7 RIM-To-Relational Database Mapping. In: IHIC Conference, Kyoto, Japan (2009) 